opening in the bladder should never be sutured; it adds a danger to the operation, for if the bladder begins to leak after the superficial wound has entirely or partly closed (Case 3), extravasation and pelvic cellulitis are likely to occur; if, on the other hand, the suturing is successful, the operation is deprived of one of its chief advantages-the free after-drainage--which is always beneficial.-London Lancet, March 18, 1893 .

\title{
ABSCESSES.
}

I. Radical and Rapid Treatment of Cold Abscess. By Dr. CALOT (Paris). The writer claims that the most voluminous and ancient cold abscesses are curable in eight days, provided that they are accessible, by means of an operative procedure in which, after entire removal of the diseased tissue, immediate union is sought for. If this be not obtained, a recurrence is nearly certain, or a fistula may persist. To succeed, the adjoined tissues must have sufficient vitality, the walls of the abscess mist not only be removed, but the surrounding tissues must be excised down deep into healthy parts. All drainage must be suppressed, for the drain renders reinfection almost certain. Even when it is aseptic it will be liable to leave a fistula that will persist indefinitely. The superficial sutures must not leave a single space, and compression must be equal and energetic and methodic. Old and extensive abscesses are especially amenable to this treatment, for the writer has thus treated with success an extensive cold abscess that has been treated in vain for nearly two years by other methods, and which contained over three quarts. $-L a$ Semainc Médicale, No. $23,1 S_{93}$.

\section{BONES, JOINTS-ORTHOPEDIC.}

I. Tuberculous Disease of the Elbow Joint. By $D_{R}$. Kosina (Goetriscex). The writer has nade a study of the material which has accumulated in the Goettingen Surgical Clinje during the last fifteen years. It consists of twenty-one cases treated 
conservatively, and II5 which were either resected or amputated. Fifty-four per cent. involved the right, and 44.5 per cent. the left arm, while only 1.5 per cent. implicated both articulations. Fortysix per cent. were males, and fifty-four were of the female sex, while in other tuberculous diseases the males usually predominate. As to etiology, in $+r .5$ per cent. tuberculosis of the skin, lungs, glands, etc., had preceded, in 12 per cent. it followed an injury, in 40.5 per cent. the origin was obscure, the patients being apparently healthy. Hereditary involvement was found in only 6 per cent. In the majority the disease began in the first twenty years of life ( 45 per cent.), but not* rarely in middle life, $i$.. , , between the twentieth and fiftieth years, while at the ages of fifty to seventy only zo per cent. was observed. Twenty-two per cent. had passed the fiftieth year. The course of the disease is chiefly very chronic, and in more than the half of the cases it comes to the formulation of fistulie, sooner or later. These appear posteriorly either between the radius and ulna, or behind the internal condyle. The affected arm is contracted in a right-angled position, and is in some cases anchylotic. The disease may attack the synovial membrane or the bone, or be from the first a mixed form. Tuberculous dropsy of the joint is much rarer than in the knee joint. Among the cases operated on $7 x$ per cent. were osteal, involving chiefly the humerus, most rarely the radius. Injection with a solution of iodoform in glycerine was, in two cases, without results; the plasterof-Paris bandage gave the same result. Resection was performed ninety-four times after Langenbeck's, and fourteen times after Koenig's method. In eight cases, on account of destructive implication of the joint, amputation was necessary. In Koenig's method the olecranon, and a large portion of the epicondyles are saved. Iodoform is dusted in and rubbed into the resected articulations, the arm placed in extension and supination for three or four weeks, before any fexion is attempted. In general, resection gives the best results, for 6o per cent. of the cases had, with more or less mobility, a serviceable arm, 33 per cent. remained anchylotic, without entirely losing the use of the member. Koenig's method is more frequently followed 
by anchylosis than that of Langenbeck. In $7+$ per cent. the operation ran an asejtic and an afebrile conrse; in 3 per cent. the result was fatal (one death from iodoform intoxication, a second from pneumonia, a third from diphtheria, and the fourth from pulmonary tuberculosis). Later, twenty-five of those operated on died from their tuberculosis in other organs; of these teri were cured of tuberculosis of the elbow joint, five retained suppurating fistula. The result in the other ten was undetermined.-Diutsche Zeitschrift fuer Clirurgic,
Bd. xxxv, Heit. I-2.

II. Treatment of Tuberculous Coxitis by Injections of Iodoform. By C. Buxi:Ek. The writer regards injections of iodoform 2.5 a step forward in the management of surgical tuberci:culosis, yet the results seem to he less farorable in tuberculous disease of the hip joint. This is due to the faulty technique. He declares Krause's method to be insufficient, and to require certain positions which, in the stage when the iodoform injections are indicated, to be difficult of execution. He recommends the following method, which has been used by Kuester for several years: One determines by palpation the place where the femoral artery crosses the pubic bone, and introduces the instrument at the inner border of the Sartorius, and on a line drawn from the femoral artery to the trochanter major, directly into the joint. At this place one can often in lean individmals feel the head of the bone, and it is indeed the shortest and most direct way into the joint. Isesion of the vessels and the crural nerve is thus aroided, as they lie more toward the median line. A series of experiments upon the cadaver lias demonstrated the advantages of this method. In all cases, on opening the joint the injected substance was not only spread over the synovial membrane, but also diffused over the head of the femur and acetabulum, $i . e_{\text {., there whe }}$ the osteal foci are usually found. Done after Krause's method the injection in some cases did not gain the joint at all, and, in the majority, was found to cover but a portion of the articulation. With this modification he hopes for better results in the treatment of tuber- 
culous coxitis. Instead of the long trocar used by Krause he prefers a hypodermic syringe containing about two and a half grams, with a tip some fire to seren centimetres in length. As an injection he uses a 20 per cent. solution of jodoform in glycerine, which he injects every eighth to fourteenth day.-Centralblalt fucr Chirurgie, No. $5 \mathrm{I}, \mathrm{r}_{9} \mathrm{~s}_{3}$.

Frask H. Prichakd (Norwalk, Ohio).

III. Hoffa's Operation for Congenital Luxation of Hip. By Dr. DexwcE. A girl, five years of age, presenting the characteristic gait of congenital luxation of the hip. The total shortening of the left lower limb was $51 / 2 \mathrm{~cm} ., 3$ of which are attributed to the riding up of the femur. There exists a primitive lumbar scoliosis with a compensation curve in the dorsal region.

An incision of $7 \mathrm{~cm}$. parallel to the axis of the great trochanter and up to $3 \mathrm{~cm}$. above it was made. The muscles were divided in the direction of the fibres of the gluteus maximus, displaying the thickened capsule. This was cut open longitudinally from one end to the other. The head of the femur was now seen, flattened from behind forward and somewhat elongated from above downward. The round ligament was missing, as well as the neck, and the head seemed directly applied on the femoral diaphysis. The anterior muscles, the pyramidal, the internal obturator and the gemelli were retracted. A cartilaginous lamella, very small, containing all the tendinous trochanter insertions, was detached from the great trochanter with a strong blunt bistoury. The head could then be drawn down by traction much better.

The cotyloid carity was small and triangular, readily adnitting the extremity of the index finger. The soft parts were removed, incision made to the bone in front, downward and backward. This detachment of the celiulo-filyrous tissue from the bone was the most intricate and delicate moment of the operation. The exposed cotyloid cavity was still too narrow to receive the femoral head. '] he lower half circumference of this cavity was enlarged with a fenestrated 
gouge, hollowing it out to increase its depth. The two cartilaginous pieces with the muscular insertions are then fixed on the head with a few catgut sutures, and all is ligated to the capsule. A sort of bridge is thus formed above the head to give it support. The capsule is sutured with catgut, leaving open only a small orifice for a thread-like drain. The skin is sutured with horse hair. A large T-shaped plaster bandage grasps the pelvis with ies horizontal branch, and winding around the lower limb down to the middle of the calf holds the thigh in abduction. Extension with five pounds of weight and counter-extension by raising the legs of the bed is employed.

The temperature rose on this evening to $3 \mathrm{~S}^{\circ} \mathrm{C}$., on the following evening to $39^{\circ} \mathrm{C}$., but it soon fell. The dressing was left in its place for about three weeks; the wound cicatrized.

The movements are free; the great trochanter is on a level with Nèlaton's line. The retraction is equal to $2 \frac{1}{2} \mathrm{~cm} .-i$. $c$., just the difference of real length between the two limbs.

Traction is continued with a weight of four pounds, massages every day; also more and more extended movements. The child still walked on crutches when she left the hospital. A year aftenvard lordosis had disappeared, but there remained a certain degree of scoliosis. The measurement of the two lower limbs gave a difference of a little less than $3 \mathrm{~cm}$.; that of the different segments of the lower limb showed nearly $1.8 \mathrm{~cm}$. against the limb operated on.Bulletin et Memoircs de la Société de Chirurgie de Paris, February, r893.

SANLEL Llotd (New York).

\section{GYNACOLOGICAL.}

I. Symphysiotomy at the Clinique Baudelocque. By A. PinaRd, M.D. (Paris). In a clinical lecture, reported in English by Dr. J. B. Henderson, the author reviews his experience with symphysiotomy during the year 1892 . Thirteen cases were subjected to operation by him and his assistants as follows : 